\title{
Economic Decision Model Suggests Total Shoulder Arthroplasty is Superior to Hemiarthroplasty in Young Patients with End-stage Shoulder Arthritis
}

\author{
Suneel B. Bhat MD, Mark Lazarus MD, Charles Getz MD, \\ Gerald R. Williams Jr MD, Surena Namdari MD, MSc
}

Received: 5 January 2016/ Accepted: 13 July 2016/Published online: 25 July 2016

(C) The Association of Bone and Joint Surgeons ( 2016

\begin{abstract}
Background Young patients with severe glenohumeral arthritis pose a challenging management problem for shoulder surgeons. Two controversial treatment options are total shoulder arthroplasty (TSA) and hemiarthroplasty. This study aims to characterize costs, as expressed by reimbursements for episodes of acute care, and outcomes associated with each treatment.

Questions/purposes We asked: for patients 30 to 50 years old with severe end-stage glenohumeral arthritis refractory to conservative management, (1) are more years of patientderived satisfactory outcome by the Neer criteria and quality-adjusted life-years (QALYs) achieved using a TSA or a hemiarthroplasty; (2) does a TSA or a hemiarthroplasty result in a greater number of revision procedures; and (3) does a TSA or a hemiarthroplasty result in greater associated costs to society?
\end{abstract}

Each author certifies that he or she, or a member of his or her immediate family, has no funding or commercial associations (eg, consultancies, stock ownership, equity interest, patent/licensing arrangements, etc) that might pose a conflict of interest in connection with the submitted article.

All ICMJE Conflict of Interest Forms for authors and Clinical Orthopaedics and Related Research ${ }^{\circledR}$ editors and board members are on file with the publication and can be viewed on request. Each author certifies that his or her institution approved or waived approval for the human protocol for this investigation and that all investigations were conducted in conformity with ethical principles of research

S. B. Bhat, M. Lazarus, C. Getz, G. R. Williams Jr, S. Namdari ( $₫)$ Rothman Institute Department of Orthopaedics, Thomas Jefferson University Hospital, 925 Chestnut Street, 5th Floor, Philadelphia, PA 19107, USA

e-mail: surena.namdari@gmail.com
Methods The incidence of glenohumeral arthritis among 30- to 50-year-old patients, outcomes, reoperation probabilities, and associated costs from TSA and hemiarthroplasty were derived from the literature. A Markov chain decision tree model was developed from these estimates with number of revisions, cost of management for patients to 70 years old as defined by reimbursement for acute-care episodes, years with "satisfactory" or "excellent" outcome by the modified Neer criteria, and QALYs gained as principle outcome measures. A Monte Carlo simulation was conducted with a cohort representing the at-risk population for shoulder arthritis between 30 and 50 years old in the United States. Results During the lifetime of a cohort of 5279 patients, hemiarthroplasty as the initial treatment resulted in 59,574 patient years of satisfactory or excellent results (11.29 per patient) and average QALYs gained of 6.55, whereas TSA as the initial treatment resulted in 85,969 patient years of satisfactory or excellent results (16.29 per patient) and average QALYs gained of 7.96. During the lifetime of a cohort of 5279 patients, a hemiarthroplasty as the initial treatment led to 2090 lifetime revisions ( 0.4 per patient), whereas a TSA as the initial treatment led to 1605 lifetime revisions ( 0.3 per patient). During the lifetime of a cohort of 5279 patients, a hemiarthroplasty as initial treatment resulted in USD 132,500,000 associated direct reimbursements (USD 25,000 per patient), whereas a TSA as initial treatment resulted in USD 125,500,000 associated direct reimbursements (USD 23,700 per patient).

Conclusions Treatment of end-stage glenohumeral arthritis refractory to conservative treatment in patients 30 to 50 years old in the United States with TSA, instead of hemiarthroplasty, would result in greater cost savings, avoid a substantial number of revision procedures, and result in greater years of satisfactory or excellent patient outcomes and greater QALYs gained. On a population 
level, TSA is the cost-effective treatment for glenohumeral arthritis in patients 30 to 50 years old.

Level of Evidence Level II, economic and decision analysis study.

\section{Introduction}

There is an increasing demand for shoulder arthroplasty in the United States population [11, 21]. According to Padegimas et al. [29], the Nationwide Inpatient Sample (NIS) showed an increase in the incidence of shoulder arthroplasty from 2002 through 2011 of $267 \%$. With everincreasing expenditures and the rising economic burden of health care, value-based reimbursement has entered the forefront of clinical decision-making [1, 6, 28, 31].

The young patient, between 30 to 50 years old, with endstage shoulder arthritis presents a unique treatment challenge given increased activity levels, higher expectations, and longer life expectancy. Owing to concern for glenoid loosening, hemiarthroplasty traditionally has been used in young patients, despite data to suggest that reoperation rates may be inferior to those of total shoulder arthroplasty (TSA), attributable to glenoid-sided wear and persistent pain [5, 14, 33, 37]. With reports of improved patient-specific satisfaction and shoulder function scores, increased longevity of implants, and widespread training, indications for shoulder arthroplasty have expanded to include younger and more-active patients. In a recent study, Padegimas et al. [29] projected an $8 \%$ yearly increase in the use of shoulder arthroplasties in patients younger than 55 years and a reduction in the relative proportion of hemiarthroplasties from $34 \%$ in 2011 to $24 \%$ of all arthroplasties by 2030 .

Evaluating treatment options for end-stage glenohumeral arthritis in younger patients poses unique challenges. As the patient population has a substantial remaining lifespan, conducting long-term prospective studies with adequate followup to capture postoperative events is challenging. Furthermore, capturing a large enough cohort to produce generalizable results often is not feasible with low-incidence conditions. Finally, traditional clinical studies often are lacking in their ability to incorporate and evaluate economic endpoints in conjunction with clinical ones during a long study period. Our approach in this study to attempt to contend with these issues was to use a Markov chain cohort decision model based on outcomes and provide transition probabilities obtained from a systematic review of the literature. This type of model allows the investigator to follow a theoretical cohort or single patient through different states of health during fixed intervals, where the patient either may stay in a given health state or transition to another one, accruing outcomes at each interval $[12,26]$. The decision model can be designed by synthesizing varied data sources, including existing clinical studies, national databases, economic data, and presenting results in a manner that combines clinical and economic elements. Decision and Markov modeling have been used increasingly in orthopaedics and have gained acceptance as a means of guiding clinical care and policy [17, 23-25, 36, 38]. Applying these methods here proves to be an effective way of exploring and understanding a challenging clinical scenario.

Specifically, we asked, for young patients with severe end-stage glenohumeral arthritis, (1) are more years with a subjective, patient-specific satisfactory outcome by the Neer criteria and quality-adjusted life-years (QALYs) achieved using a TSA or a hemiarthroplasty; (2) does a TSA or a hemiarthroplasty result in a greater number of revision procedures; and (3) does a TSA or a hemiarthroplasty result in higher associated costs, as expressed by physician and facility reimbursement during surgical episodes of care?

\section{Materials and Methods}

\section{Model Design}

Our decision analysis was conducted with Monte Carlo simulation with a cycle length of 1 year for a cohort representative of the US population distribution using TreeAge Pro (TreeAge Pro 2013; TreeAge Software Inc, Williamstown, MA, USA). Monte Carlo simulations, or stochastic simulations, rely on repeat sampling of probabilities to obtain distributions of outcomes. Using this method with a Markov chain decision model allows simulation of individual patients across the model, incorporating variability at each point of probability and providing representative cohort-wide outcomes [22].

A population cohort between 30 to 50 years 6ld was chosen based on incidence rates for TSA and hemiarthroplasty derived from the NIS [20].

Our decision model consisted of two primary treatment arms. Patients with glenohumeral arthritis could undergo either primary TSA (humeral head replacement and allpolyethylene glenoid resurfacing) or hemiarthroplasty (humeral head replacement only). At every annual cycle in the model, there was an age-specific risk of mortality based on United States life tables [39]. Intraoperative and perioperative mortality were assumed to be similar and negligible for the two procedures and so were not included.

Patients who initially had a TSA and were alive at each interval of the model remained in their current state (TSA), underwent a revision TSA, or underwent a 
hemiarthroplasty. Patients with a revision TSA either remained in that state (revision TSA) or underwent a hemiarthroplasty or a second revision TSA. Patients with a revision hemiarthroplasty remained in that state (revision hemiarthroplasty) or underwent a revision TSA. Patients initially treated with hemiarthroplasty who remained alive at each interval of the model either remained in that state (hemiarthroplasty) or underwent revision TSA. If they had revision surgery, they remained in that state (revision TSA) or had a second revision TSA or revision hemiarthroplasty (Fig. 1).

Given the age of our cohort, a maximum of two revisions was allowed before the patient was assumed to drop out of the model or undergo a salvage procedure (reverse shoulder arthroplasty, resection arthroplasty, or fusion). Given limited data regarding the probability of conversion of hemiarthroplasty or TSA to reverse arthroplasty, resection arthroplasty, or fusion, these procedures were not included in the model and 70 years was selected as the upper age limit of the model.
Reimbursement as a Proxy for Episode of Care Costs

Estimated reimbursement for hemiarthroplasty was calculated using maximum Medicare reimbursement of Diagnosis Related Group (DRG) code 508 (Major Shoulder or Elbow Joint Procedures W/O CC/MCC) for hospital fees, Current Procedural Terminology (CPT) code 23470 (hemiarthroplasty) for surgeon fees, and CPT code 1638 (Anesthesia for TSA) with 10 base units and four time units for anesthesia (Table 1). Estimated reimbursement for TSA was calculated using estimated maximum Medicare reimbursement of DRG code 508 for hospital fees, CPT code 23472 (TSA) for surgeon fees, and CPT code 1638 with 10 base units and six time units for anesthesia fees. Facility reimbursements were calculated using Inpatient Medicare Price and average Medicare physician reimbursements obtained from the 2014 Centers for Medicare \& Medicaid Services (CMS) Physician Fee Schedule using the CMS Physician Fee Schedule Search [8]. All Medicare reimbursement figures for the initial episode of care and

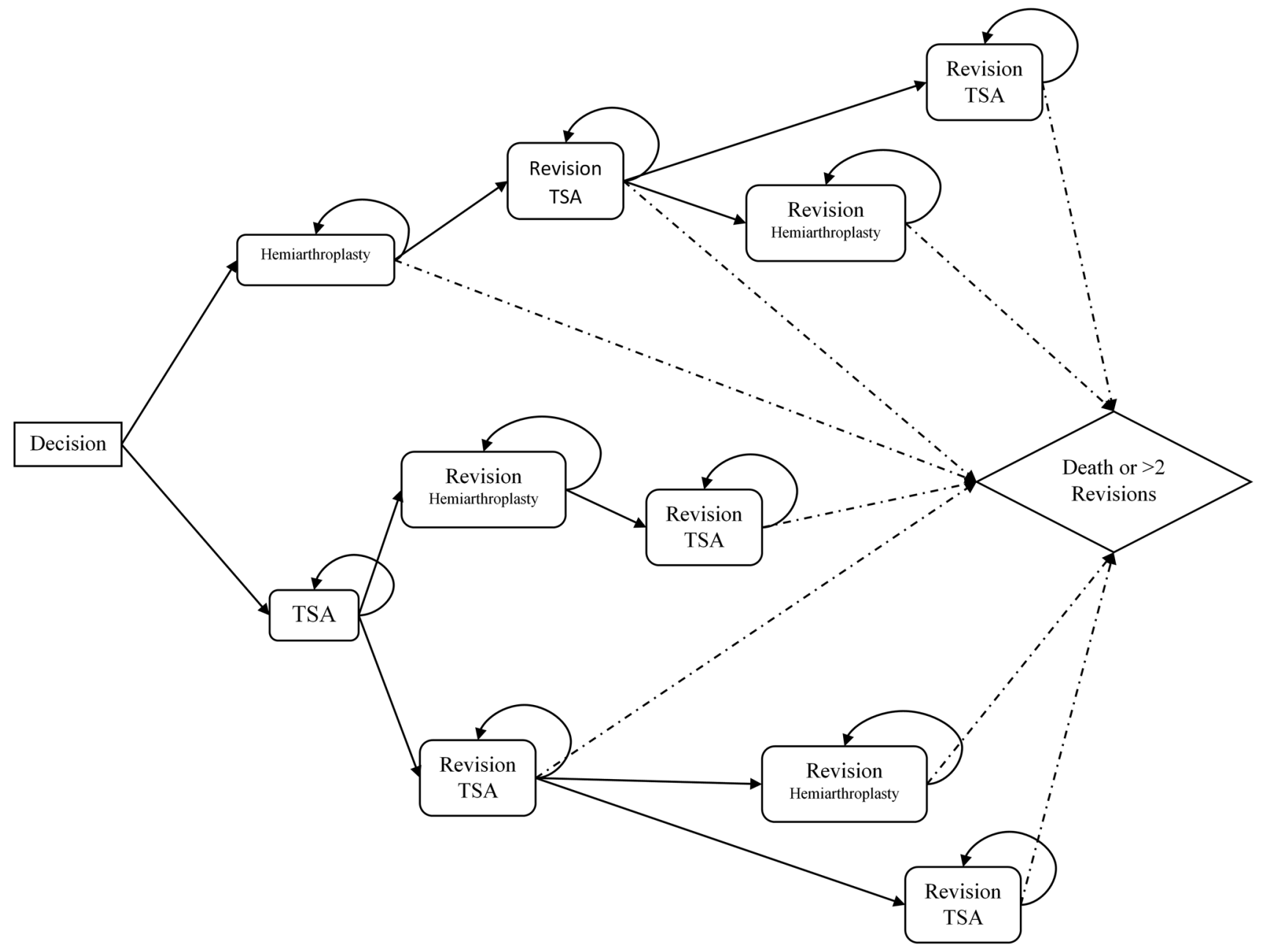

Fig. 1 A schematic of the Markov decision model is shown. TSA = total shoulder arthroplasty. 
Table 1. Costs

\begin{tabular}{lll}
\hline Category & TSA/revision TSA & Hemiarthroplasty/revision HA \\
\hline DRG & 508 & 508 \\
Facility reimbursement & USD 11,307.89 & USD 11,307.89 \\
CPT code & 23472 & 23470 \\
Surgeon reimbursement & USD 1499.18 & USD 1235.89 \\
Anesthesia CPT code & 1638 & 1638 \\
Anesthesia base units & 10 & 10 \\
Anesthesia time units & 6 & 4 \\
Anesthesia reimbursement & USD 351.04 & USD 307.16 \\
Total Medicare reimbursement & USD 13,158.11 & USD 12,850.94 \\
Estimated private reimbursement & USD 18,289.77 (Sensitivity analysis range, & USD 17,892.81 (Sensitivity analysis range, \\
& USD 16,461-USD 20,119) & USD 16,104-USD 19,682) \\
\hline
\end{tabular}

TSA = total shoulder arthroplasty; HA = hemiarthroplasty; DRG = Diagnosis Related Group; CPT = Current Procedural Terminology.

subsequent revisions were converted to estimates of private reimbursement rates with a conversion factor of 1.39 , based on the average conversion factor from a national survey, a figure used in prior economic analyses [19, 23]. The differences in implant costs are not reflected in this analysis as we took the economic perspective of society and payers, and variability in implant costs are absorbed by hospitals as part of operating costs and not directly seen by patients, insurance companies, or the CMS.

\section{Survivorship and Outcomes}

Survivorship rates for TSA and hemiarthroplasty among patients younger than 50 years with end-stage glenohumeral arthritis were obtained from the literature (Table 2) $[2-4,7,9,13,15,37]$. For each procedure, the survivorship rate was calculated by performing a systematic review following PRISMA (Preferred Reporting Items for Systematic Reviews and Meta-Analyses) guidelines. PubMed and Scopus computerized literature databases were searched through July 2015. Articles were identified by searches using relevant keywords ("shoulder hemiarthroplasty", "total shoulder arthroplasty", "glenohumeral hemiarthroplasty", "glenohumeral total shoulder arthroplasty"). For primary arthroplasty in young patients, studies were included if they were (1) written in English, (2) contained a minimum of 50 patients at baseline, (3) included only patients younger than 50 years, and (4) reported survivorship at 10 years of followup. One study by Sperling et al. [37] qualified for inclusion, and annual incremental failure rates for primary hemiarthroplasty and TSA were derived from 10-year estimates in patients 50 years or younger, which vary from an annual failure rate of 0.008 to 0.0125 based on time from surgery. The probability of subsequent revision surgery was determined by performing systematic reviews of the literature for each procedure. PubMed and Scopus computerized literature databases were searched through July 2015 using relevant keywords ("revision shoulder hemiarthroplasty", "revision total shoulder arthroplasty", "revision glenohumeral hemiarthroplasty", "revision glenohumeral total shoulder arthroplasty"). Articles were identified by searches using relevant keywords (Table 2). Studies were included if they were (1) written in English, (2) contained a minimum of 10 patients at baseline, (3) reported rates of revision and described the type of revision performed, and (4) included a minimum of 2 years of clinical followup. Based on these criteria and using pooled statistics, the probability of revision to either hemiarthroplasty or revision TSA after TSA failure was derived from four case series describing the respective revision procedures $[2,9,13,15]$. Subsequent probability of revision of a revision hemiarthroplasty was derived from these same studies $[2,9,13,15]$. Probability of revision of a revision TSA was derived from six retrospective case series $[2,4,7,9,13,15]$. The years with a "satisfactory" or "excellent" result for each procedure were derived from studies that reported the modified Neer rating [2, 7, 9, 37]. For the modified Neer rating, to be considered as having an excellent result, patients had to be satisfied with the procedure and have at least $45^{\circ}$ external rotation, active abduction of at least $140^{\circ}$, and no or slight pain $[10,27,37]$. Patients were considered to have a satisfactory result if they were satisfied with the procedure and had no, slight, or moderate pain only with vigorous activities; at least $20^{\circ}$ external rotation; and active abduction of at least $90^{\circ}$. The result was unsatisfactory if any of the criteria were not met. 
Table 2. Base case parameters

\begin{tabular}{|c|c|c|}
\hline Parameter & Probability & Sensitivity analysis range \\
\hline Annual failure rate of primary hemiarthroplasty $0-10$ years & $0.018[37]$ & \\
\hline Annual failure rate of primary hemiarthroplasty $11-20$ years & $0.007[37]$ & \\
\hline Annual failure rate of primary hemiarthroplasty $21-30$ years & $0.0125[37]$ & \\
\hline Annual failure rate of primary hemiarthroplasty $31-40$ years & $0.0125[37]$ & \\
\hline Annual failure rate of primary TSA $0-10$ years & $0.003[37]$ & \\
\hline Annual failure rate of primary TSA $11-20$ years & $0.013[37]$ & \\
\hline Annual failure rate of primary TSA $21-30$ years & $0.008[37]$ & \\
\hline Annual failure rate of primary TSA $31-40$ years & $0.008[37]$ & \\
\hline Proportion revised to hemiarthroplasty after primary TSA & $0.482[2,9,13,15]$ & $0.434-0.530$ \\
\hline Proportion revised to TSA after primary TSA & $0.518[2,9,13,15]$ & $0.466-0.570$ \\
\hline Annual rate of revision hemiarthroplasties for failed revision TSA after hemiarthroplasty & $0.0116[2,7,9,13,15]$ & $0.010-0.013$ \\
\hline Annual rate of revision TSAs for failed revision TSA after hemiarthroplasty & $0.0124[2,7,9,13,15]$ & $0.011-0.014$ \\
\hline Annual rate of revision TSAs for failed revision hemiarthroplasty after primary TSA & $0.031[2,9,13]$ & $0.028-0.034$ \\
\hline Annual rate of resection for failed revision hemiarthroplasties after primary TSA & $0.0083[2,9,13]$ & $0.007-0.009$ \\
\hline Annual rate of revision hemiarthroplasties for failed revision TSA after primary TSA & $0.0103[2,4,9,13]$ & $0.009-0.011$ \\
\hline Annual rate of revision TSAs for failed revision TSA after primary TSA & $0.0042[2,4,9,13]$ & $0.004-0.005$ \\
\hline Annual rate of reverse TSAs for failed revision TSA after primary TSA & $0.023[2,4,9,13]$ & $0.021-0.025$ \\
\hline Annual probability of satisfaction after primary TSA & $0.62[37]$ & $0.558-0.682$ \\
\hline Annual probability of satisfaction after primary hemiarthroplasty & $0.4[37]$ & $0.36-0.44$ \\
\hline Annual probability of satisfaction after revision hemiarthroplasty after primary TSA & $0.189[2,9]$ & $0.17-0.208$ \\
\hline Annual probability of satisfaction after revision TSA for primary hemiarthroplasty & $0.533[7]$ & $0.480-0.586$ \\
\hline Annual probability of satisfaction after revision TSA for primary TSA & $0.381[2,9]$ & $0.343-0.419$ \\
\hline
\end{tabular}

$\mathrm{TSA}=$ total shoulder arthroplasty.

\section{Utilities}

Health-related quality-of-life measures are traditionally a component of a cost-effectiveness analysis and are estimates that attempt to capture the relative health experience of a patient compared with a state of normal health. Utilities used to calculate QALYs are theoretical estimates derived from health questionnaires and opinion. A utility of 0 represents the value of being dead, while a utility of 1 represents perfect health and function. In disease processes or treatments without accurate quality-of-life assessments, calculating utilities is particularly challenging. A prior cost-effectiveness study evaluating TSA and hemiarthroplasty for glenohumeral arthritis was used as precedent for utilities in this study, which derived utilities from preference-based patient measure of other arthritic conditions [24]. A value of 0.6 was used as the baseline for glenohumeral arthritis. Utility values of 0.9 for TSA and 0.85 for hemiarthroplasty were used, and 0.8 was used for a revision hemiarthroplasty or TSA from a primary TSA and 0.82 was used for revision TSA from a primary hemiarthroplasty. Revision procedures were assigned a one-time disutility of -0.02 . We approximated incremental QALYs from the years spent in each state in the Markov model and respective utilities, and calculated the incremental costeffectiveness ratio (ICER) for both treatments. Utilities subsequently were varied in the sensitivity analysis to evaluate robustness over a range of utility scores.

\section{Statistical Analysis}

The annual cohort size of patients 30 to 50 years old with early glenohumeral arthritis requiring surgery was estimated from the NIS to be 5279 patients. Individual simulations were run with 5279 iterations, which adequately achieves a stable estimate of the central tendency, however simulations for each set of conditions were repeated 10 times and the results averaged to capture the occurrence of rare events without artificially inflating the sample size [20,30]. Results are presented with $95 \%$ CIs to reflect and characterize stochastic variability in model simulations and uncertainty in differences in comparative estimates between cohorts; these do not necessarily reflect parameter uncertainties, which were further characterized with a sensitivity analysis.

Deterministic and probabilistic sensitivity analyses were conducted to determine robustness of our model and the 
effect of varying key variables. To test the effect of age at its extremes, the simulation was repeated with a cohort of 30-year-old-only patients One-way sensitivity analysis to identify break-even thresholds were performed infinitely varying costs of hemiarthroplasty for costs, and repeated varying the 10-year survival rate of hemiarthroplasty for revisions. Finally a probabilistic sensitivity analysis of all nontime-dependent variables including point probabilities, outcome measures, and costs was conducted across a uniform distribution of values (Table 2).

\section{Results}

\section{Years of Satisfactory Outcome}

By the modified Neer criteria, hemiarthroplasty as the initial treatment resulted in 59,574 total satisfactory or excellent patient years compared with 85,969 years for total TSA ( $p<0.001$ ) (Fig. 2). Use of primary TSA in all cases of early glenohumeral arthritis would result in 26,395 more years with a satisfactory or excellent result (95\% CI, 23,529-29,260) compared with hemiarthroplasty during the lifetime of the cohort. TSA provided average QALYs gained of 7.96 (95\% CI, 7.77-8.15) whereas hemiarthroplasty provided average QALYs gained of 6.55 (95\% CI, 6.36-6.74) during the lifetime of the cohort against a base-case scenario of end-stage glenohumeral arthritis. A TSA for early glenohumeral arthritis represented an incremental QALY gain of 1.41 relative to hemiarthroplasty (95\% CI, 1.37-1.679; p < 0.001). Probabilistic sensitivity analysis showed eight QALYs and 16.23 satisfied years gain per-patient in the TSA arm, and 6.66 QALYs and 11.24 satisfied years gain for hemiarthroplasty, with TSA remaining favorable with an incremental QALY gain of 1.35 over hemiarthroplasty (95\% CI, 1.08-1.62; p < 0.001) and an incremental gain of five satisfied years (95\% CI, 4.4-5.5; p < 0.001), supporting the robustness of the model results,

\section{Number of Revision Procedures}

Hemiarthroplasty as the initial preferred treatment resulted in 2090 revisions compared with 1605 revisions when TSA was the initial treatment choice ( $p<0.001)$ (Fig. 3). A primary TSA in all cases of early glenohumeral arthritis would avoid 486 revisions (95\% CI, 363-609) compared with hemiarthroplasty. Probabilistic sensitivity analysis showed a lifetime per-patient revision rate of 0.31 for TSA and 0.4 for hemiarthroplasty, with a TSA avoiding on average 0.09 revisions (95\% CI, 0.07-0.11; $\mathrm{p}<0.001)$

\section{Economic Analysis}

During the lifetime of the cohort, total reimbursements were USD 132,500,000 when hemiarthroplasty was selected as the initial treatment and USD 126,500,000 when TSA was selected ( $p<0.001$ ) (Fig. 4). A primary TSA in all cases of early glenohumeral arthritis would save USD 6,900,000 (95\% CI, USD 4,600,000-USD 9,100,000) compared with hemiarthroplasty. The ICER calculated for hemiarthroplasty compared with a base-case scenario of glenohumeral arthritis was USD 3832 per QALY and the ICER calculated for TSA compared with a base-case scenario of glenohumeral arthritis was USD 2989 per QALY. Both showed cost-effectiveness compared with the conservative traditional threshold of USD 50,000 per QALY, with TSA proving to be the superior cost-effective strategy [40].

\section{Sensitivity Analysis}

Our probabilistic sensitivity analysis showed an average ICER of USD 3795 per QALY for a hemiarthroplasty and USD 2985 per QALY for a TSA. A scatterplot of lifetime incremental QALYs and the associated incremental lifetime reimbursement showed that nearly all cases are superior to an accepted cost-effectiveness threshold of USD 50,000 per QALY, with cases with an initial TSA distributed in a more dominant manner (toward the bottom right of the graph) (Fig. 5). Cases are most strongly clustered by the number of revisions, suggesting that this variable is the most important driver of cost-effectiveness in our model.

Threshold analysis showed a cohort consisting only of 30-year-old patients would continue to support the use of TSA over hemiarthroplasty on a population level. Although the differences in cost of reimbursement (USD 2,700,000 versus USD 6,900,000) and lifetime revisions (264 versus 486) were less substantial than those seen for the 30- to 50year-old cohort, there was a greater difference in the number of years with a satisfactory or excellent result (32,782 versus 26,395$)$. To reach cost equivalence with a TSA, threshold analysis of reimbursements identified that hemiarthroplasty would have to be reimbursed at less than USD 16,600 (compared with USD 17,900) (Fig. 6). To achieve a similar number of lifetime revisions compared with a TSA, the 10-year survival rate of hemiarthroplasty would need to increase to $91 \%$ (from $82 \%$ ) (Fig. 7).

\section{Discussion}

A previous cost-effectiveness analysis, using a Markov decision analysis, suggested that TSA is more clinically 
Fig. 2 The total lifetime patient years with a satisfactory or excellent result are shown for treatment of glenohumeral arthritis in patients 30 to 50 years old with hemiarthroplasty versus TSA, as determined using the modified Neer criteria. TSA $=$ total shoulder arthroplasty.

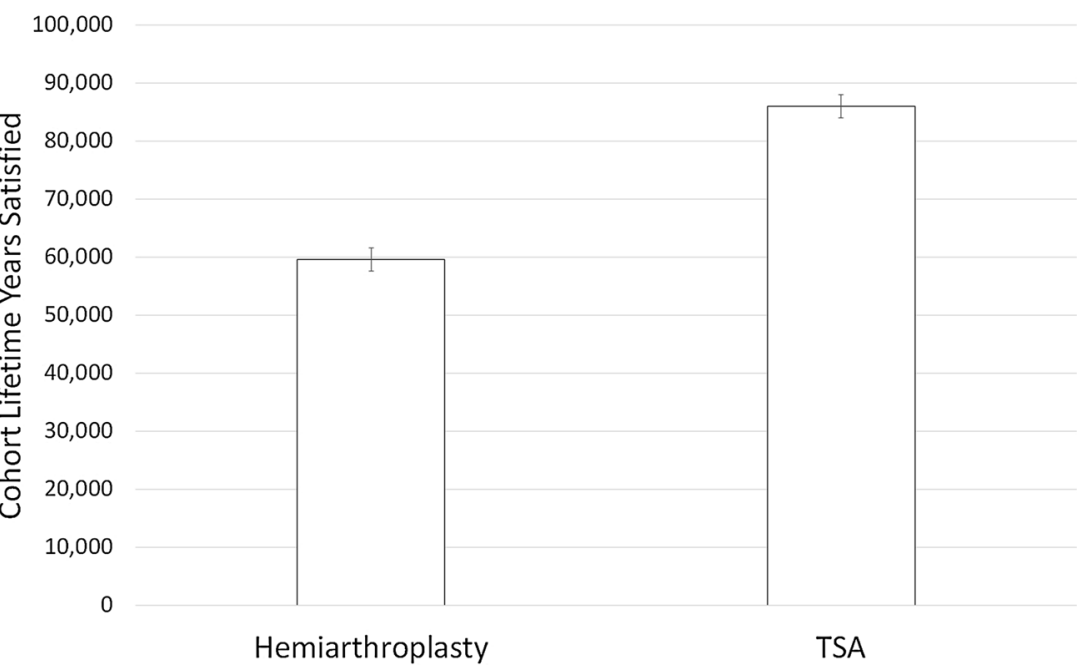

Fig. 3 The total lifetime revisions for treatment of glenohumeral arthritis in patients 30 to 50 years old with hemiarthroplasty versus TSA are shown. TSA = total shoulder arthroplasty.

Fig. 4 The total lifetime cost in dollars for treatment of patients 30 to 50 years old with hemiarthroplasty versus TSA is shown. TSA $=$ total shoulder arthroplasty.
2500

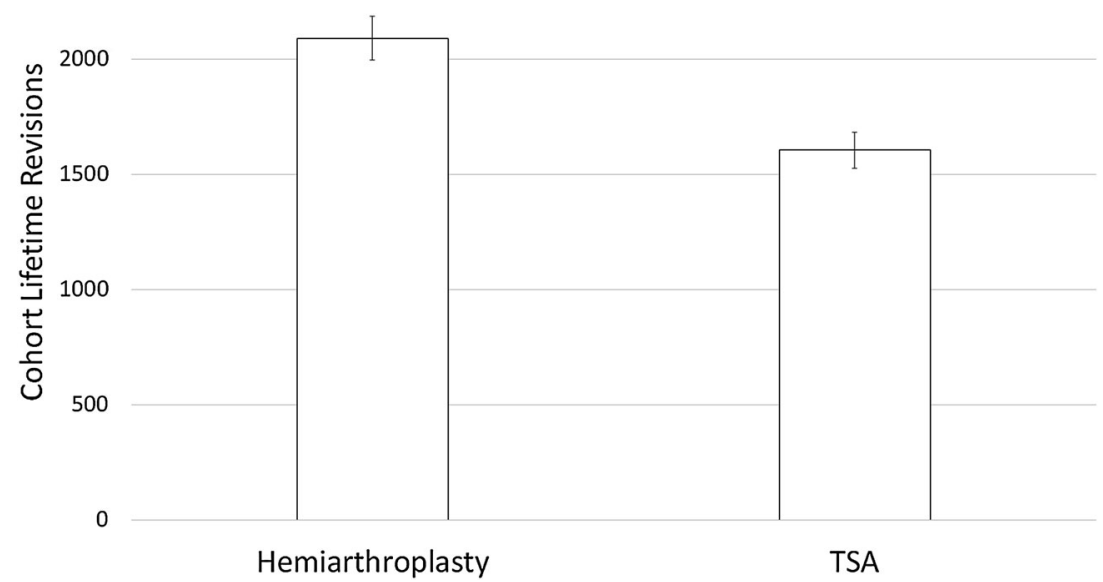

$136,000,000$

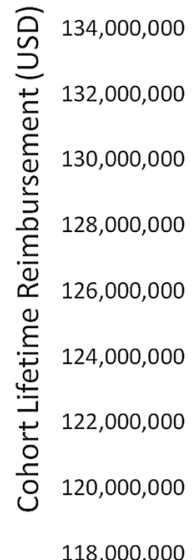

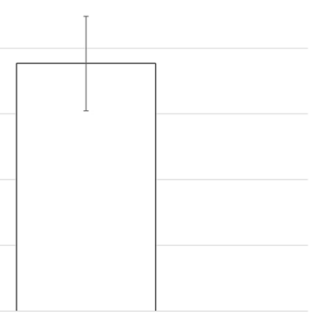

TSA 
Fig. 5 The incremental lifetime reimbursements and incremental QALYs of TSA and hemiarthroplasty each compared with no treatment as determined by probabilistic sensitivity analysis are shown. TSA $=$ total shoulder arthroplasty.
Fig. 6 The one-way sensitivity analysis with varying reimbursement of primary hemi arthroplasty is shown.
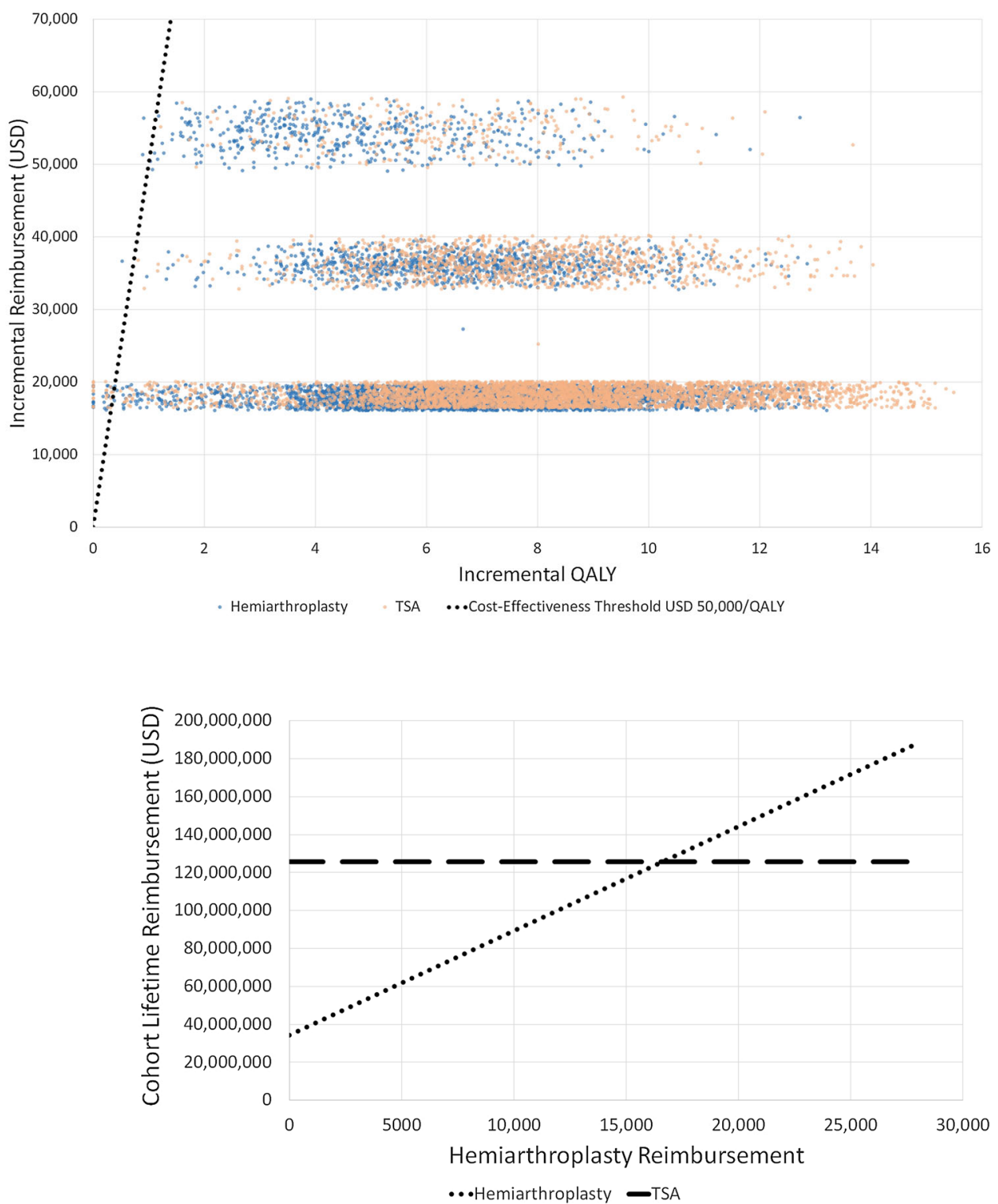

effective and less costly than hemiarthroplasty for patients 64 years or older [24]. To our knowledge, an economicbased decision model has not been performed for young patients with severe glenohumeral arthritis. Based on probabilities derived from studies identified in a systematic review, annual procedure volumes from a large national database, and reimbursement estimates derived from Medicare rates, our study showed that TSA provides more satisfactory or excellent patient years, greater QALYs gained, fewer revisions, and lower cost compared with hemiarthroplasty in the treatment of end-stage glenohumeral arthritis in the young patient.

This study has several limitations. In particular, we relied on estimates from systematic reviews for our model, and thereby any inherent weaknesses in these referenced studies translated to weaknesses in our cost-effectiveness analysis. For example, revision rates may improve for TSA or for hemiarthroplasty with improved glenoid fixation or alternative bearing surfaces, respectively. Similarly, the effect of morphologic features of the glenoid on revision rates could not be adequately modeled in this study and may represent a specific clinical scenario where hemiarthroplasty may be preferred. In addition, we could not control for differences in surgeon experience on outcomes because the failure rates and outcome measures used to create the inputs in our analysis all come from high-volume centers [24]. This is important to keep in mind, as our analysis would remain true only for a skilled shoulder surgeon who performs a high-enough volume of each procedure to be facile in both. Given the results of our 
Fig. 7 A one-way sensitivity analysis with varying survival rate of primary hemiarthroplasty is shown.

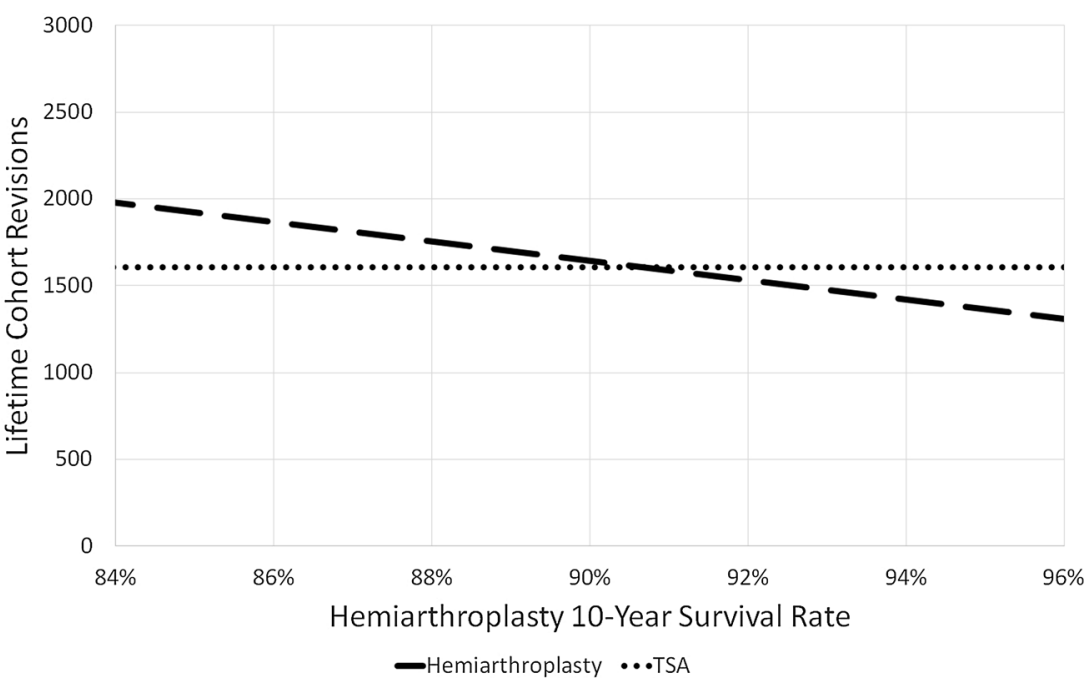

study, we recommend glenohumeral arthritis in younger patients be treated only by surgeons skilled in TSA and hemiarthroplasty to provide the optimal care for these patients. Given that a TSA is more technically demanding than hemiarthroplasty, it is possible that survival rates, outcomes, and the results of our analysis would be different if performed at lower-volume centers. In terms of the actual design of the model, utilities typically are used in cost-effectiveness analyses as measures of quality of life and are used to generate QALYs. Given the absence of studies that reported measures of utilities in shoulder osteoarthritis or arthroplasty in young patient populations, we evaluated shoulder-specific outcome based on the modified Neer rating in addition to utilities derived from a study of older patients [24]. We used the QALY values derived from the cost-effectiveness study of Mather et al. [24], which were derived from aggregate analysis of the hip and knee arthroplasty literature. Finally, although conversion of hemiarthroplasty or TSA to reverse arthroplasty continues to become a more common treatment for failed primary arthroplasties, data regarding conversion rates to reverse arthroplasty and outcomes are limited. As a result, reverse arthroplasty was considered a salvage procedure in this model. As further data regarding the use of reverse arthroplasty in cases of failed hemiarthroplasty or TSA become known, this model can be refined and made more comprehensive. Finally, the use of reimbursement data as a proxy for total costs of care may not reflect total costs, as it cannot incorporate lost wages or other economic output, medical costs outside the episode of care, or intangible and indirect costs of disability. However, because these external costs presumably would be equivalent or proportional for the two treatment arms, our findings may understate the dominance of TSA over hemiarthroplasty.

Our model showed that a TSA for glenohumeral joint osteoarthritis in young patients leads to greater number of years of satisfactory or excellent outcomes. This was not surprising given multiple studies that showed better functional results and pain relief with TSA compared with hemiarthroplasty $[3,5,16,33,35]$. In a Cochrane review, Singh et al. [35] showed that patients who underwent hemiarthroplasty compared with TSA had significantly worse American Shoulder and Elbow Surgeon (ASES) scores and nonsignificantly worse pain and Western Ontario Osteoarthritis Shoulder scores. In a study of 46 TSAs and 20 hemiarthroplasties, Bartelt et al. [3] reported significantly less pain, greater active elevation, and higher satisfaction in patients undergoing TSA compared hemiarthroplasty at a mean of 7 years.

Our statistical model and simulation suggest that routine use of TSA in young patients with glenohumeral joint osteoarthritis would lead to greater overall years of implant survival compared with hemiarthroplasty. Data regarding survival of hemiarthroplasty and TSA were obtained from a systematic review specific to young patients. Sperling et al. [37] reviewed results of 78 hemiarthroplasties and 36 TSAs in patients younger than 50 years and reported survival rates of $82 \%$ and $97 \%$ for hemiarthroplasty and TSA at 10 years, respectively. In a more-recent study with mixed arthroplasty designs, Bartelt et al. [3] reported $92 \%$ survival at 10 years for TSA and $72 \%$ for hemiarthroplasty in patients younger than 55 years. Because this was retrospective nonrandomized data, it was possible that young patients who underwent hemiarthroplasty were more-active and higher-demand patients than those who underwent TSA, possibly influencing survival. Although on a population level, the TSA showed superior survival compared with a hemiarthroplasty, case-by-case variability based on activity, work demands, expectations, and patient compliance must be considered. Additionally, it is possible that nonprosthetic options on the glenoid side such as ream and run $[16,18]$ or alternative bearing surfaces on the humeral 
side could result in improved survival and patient-specific outcome with hemiarthroplasty.

In this study, we used Medicare reimbursement data to determine the overall cost to health system payers, consistent with the US Panel for Cost-Effectiveness in Health and Medicine recommendation for a societal perspective in economic analysis [24, 32, 34, 36]. This did not include implant costs, other direct costs, or indirect costs to the provider or hospital system. Mather et al. [24] showed that in a cohort of patients who were 64 years old, a TSA would need to cost at least USD 2237 more than hemiarthroplasty to become cost neutral. Similarly, we showed that in a cohort of young patients, a TSA would need to cost at least USD 1690 more than hemiarthroplasty to achieve cost equivalence. Even if the reimbursement for hemiarthroplasty and TSA was equivalent, effectiveness, in terms of number of years with a satisfactory or excellent result, would continue to favor TSA. For every 1000 hemiarthroplasties performed for glenohumeral osteoarthritis in patients between 30 and 50 years old, the health system would save USD 1,309,369 and gain 5000 years with a satisfactory or excellent result if a TSA had been performed instead. The projected differences in reimbursement are important for physicians, policymakers, healthcare payers, hospital administrators, and implant manufacturers to consider in decision-making.

Our study showed that at the point when surgery is being considered after exhausting nonoperative treatment options, treating end-stage glenohumeral arthritis in patients 30 to 50 years old in the United States with TSA instead of hemiarthroplasty would result in greater cost savings, avoid a substantial number of revision procedures, result in greater years of a satisfactory or excellent patient outcome, and greater QALYs gained. Because utility values for shoulder arthroplasty in this patient population do not exist, and because the data on outcomes of hemiarthroplasty and TSA in young patients are limited to Level III and IV studies, further research is necessary to confirm these findings. On a population level, we conclude that the TSA is the cost-effective surgical option for recalcitrant operative end-stage glenohumeral arthritis in patients 30 to 50 years old.

\section{References}

1. Alphs Jackson H, Walsh B, Abecassis M. A surgeon's guide to bundled payment models for episodes of care. JAMA Surg. 2016;151:3-4.

2. Antuna SA, Sperling JW, Cofield RH, Rowland CM. Glenoid revision surgery after total shoulder arthroplasty. J Shoulder Elbow Surg. 2001;10:217-224.

3. Bartelt R, Sperling JW, Schleck CD, Cofield RH. Shoulder arthroplasty in patients aged fifty-five years or younger with osteoarthritis. J Shoulder Elbow Surg. 2011;20:123-130.
4. Bonnevialle N, Melis B, Neyton L, Favard L, Molé D, Walch G, Boileau P. Aseptic glenoid loosening or failure in total shoulder arthroplasty: revision with glenoid reimplantation. J Shoulder Elbow Surg. 2013;22:745-751.

5. Bryant D, Litchfield R, Sandow M, Gartsman GM, Guyatt G, Kirkley A. A comparison of pain, strength, range of motion, and functional outcomes after hemiarthroplasty and total shoulder arthroplasty in patients with osteoarthritis of the shoulder: a systematic review and meta-analysis. J Bone Joint Surg Am. 2005;87:1947-1956.

6. Burwell SM. Setting value-based payment goals: HHS efforts to improve U.S. health care. N Engl J Med. 2015;372:897-899.

7. Carroll RM, Izquierdo R, Vazquez M, Blaine TA, Levine WN, Bigliani LU. Conversion of painful hemiarthroplasty to total shoulder arthroplasty: long-term results. J Shoulder Elbow Surg. 2004;13:599-603.

8. Centers for Medicare \& Medicaid Services. Physician Fee Schedule Look-up Tool. Available at: https://www.cms.gov/ Medicare/Medicare-Fee-for-Service-Payment/PFSLookup/index. html?redirect=/pfslookup/. Accessed June 27, 2016.

9. Cheung EV, Sperling JW, Cofield RH. Revision shoulder arthroplasty for glenoid component loosening. J Shoulder Elbow Surg. 2008;17:371-375.

10. Cofield RH. Total shoulder arthroplasty with the Neer prosthesis. J Bone Joint Surg Am. 1984;66:899-906.

11. Day JS, Lau E, Ong KL, Williams GR, Ramsey ML, Kurtz SM. Prevalence and projections of total shoulder and elbow arthroplasty in the United States to 2015. J Shoulder Elbow Surg. 2010;19:1115-1120.

12. Detsky AS, Naglie G, Krahn MD, Redelmeier DA, Naimark D. Primer on medical decision analysis: Part 2. Building a tree. Med Decis Making. 1997;17:126-135.

13. Deutsch A, Abboud JA, Kelly J, Mody M, Norris T, Ramsey ML, Iannotti JP, Williams GR. Clinical results of revision shoulder arthroplasty for glenoid component loosening. J Shoulder Elbow Surg. 2007;16:706-716.

14. Dillon MT, Inacio MCS, Burke MF, Navarro RA, Yian EH. Shoulder arthroplasty in patients 59 years of age and younger. $J$ Shoulder Elbow Surg. 2013;22:1338-1344.

15. Dines JS, Fealy S, Strauss EJ, Allen A, Craig EV, Warren RF, Dines DM. Outcomes analysis of revision total shoulder replacement. J Bone Joint Surg Am. 2006;88:1494-1500.

16. Duan X, Zhang W, Dong X, Liu M, Gao Y, Huang F, Li J, Xiang Z. Total shoulder arthroplasty versus hemiarthroplasty in patients with shoulder osteoarthritis: a meta-analysis of randomized controlled trials. Semin Arthritis Rheum. 2013;43:297-302.

17. Ghomrawi HM, Eggman AA, Pearle AD. Effect of age on costeffectiveness of unicompartmental knee arthroplasty compared with total knee arthroplasty in the U.S. J Bone Joint Surg Am. 2015;97:396-402.

18. Gilmer BB, Comstock BA, Jette JL, Warme WJ, Jackins SE, Matsen FA. The prognosis for improvement in comfort and function after the ream-and-run arthroplasty for glenohumeral arthritis: an analysis of 176 consecutive cases. J Bone Joint Surg Am. 2012;94:e102.

19. Ginsburg PB. Wide variation in hospital and physician payment rates evidence of provider market power. Res Brief. 2010;16: $1-11$.

20. HCUP National Inpatient Sample (NIS). Healthcare Cost and Utilization Project (HCUP). Available at: https://www.hcup-us. ahrq.gov/nisoverview.jsp. Accessed June 27, 2016.

21. Kim SH, Wise BL, Zhang Y, Szabo RM. Increasing incidence of shoulder arthroplasty in the United States. J Bone Joint Surg Am. 2011;93:2249-2254.

22. Krahn MD, Naglie G, Naimark D, Redelmeier DA, Detsky AS. Primer on medical decision analysis: Part 4. Analyzing the model 
and interpreting the results. Med Decis Making. 1997;17:142151.

23. Mather RC 3rd, Koenig L, Acevedo D, Dall TM, Gallo P, Romeo A, Tongue J, Williams G Jr. The societal and economic value of rotator cuff repair. J Bone Joint Surg Am. 2013;95:1993-2000.

24. Mather RC 3rd, Watters TS, Orlando LA, Bolognesi MP, Moorman CT 3rd. Cost effectiveness analysis of hemiarthroplasty and total shoulder arthroplasty. J Shoulder Elbow Surg. 2010;19:325-334.

25. Mostafavi Tabatabaee R, Rasouli MR, Maltenfort MG, Parvizi J. Cost-effective prophylaxis against venous thromboembolism after total joint arthroplasty: warfarin versus aspirin. J Arthroplasty. 2015;30:159-164.

26. Naimark D, Krahn MD, Naglie G, Redelmeier DA, Detsky AS. Primer on medical decision analysis: Part 5. Working with Markov processes. Med Decis Making. 1997;17:152-159.

27. Neer CS 2nd, Watson KC, Stanton FJ. Recent experience in total shoulder replacement. J Bone Joint Surg Am. 1982;64:319-337.

28. Oberlander J, Laugesen MJ. Leap of faith: Medicare's new physician payment system. N Engl J Med. 2015;373:1185-1187.

29. Padegimas EM, Maltenfort M, Lazarus MD, Ramsey ML, Williams GR, Namdari S. Future patient demand for shoulder arthroplasty by younger patients: national projections. Clin Orthop Relat Res. 2015;473:1860-1867.

30. Petrou S, Gray A. Economic evaluation using decision analytical modelling: design, conduct, analysis, and reporting. BMJ. 2011;342:d1766.

31. Rosenthal MB. Physician payment after the SGR: the new meritocracy. N Engl J Med. 2015;373:1187-1189.

32. Russell LB, Gold MR, Siegel JE, Daniels N, Weinstein MC. The role of cost-effectiveness analysis in health and medicine. Panel on Cost-Effectiveness in Health and Medicine. JAMA. 1996;276:1172-1177.

33. Sandow MJ, David H, Bentall SJ. Hemiarthroplasty vs total shoulder replacement for rotator cuff intact osteoarthritis: how do they fare after a decade? J Shoulder Elbow Surg. 2013;22:877885 .

34. Siegel JE, Weinstein MC, Russell LB, Gold MR. Recommendations for reporting cost-effectiveness analyses. Panel on CostEffectiveness in Health and Medicine. JAMA. 1996;276:13391341.

35. Singh JA, Sperling J, Buchbinder R, McMaken K. Surgery for shoulder osteoarthritis: a Cochrane systematic review. $J$ Rheumatol. 2011;38:598-605.

36. Slover J, Espehaug B, Havelin LI, Engesaeter LB, Furnes O, Tomek I, Tosteson A. Cost-effectiveness of unicompartmental and total knee arthroplasty in elderly low-demand patients: a Markov decision analysis. J Bone Joint Surg Am. 2006;88:23482355.

37. Sperling JW, Cofield RH, Rowland CM. Minimum fifteen-year follow-up of Neer hemiarthroplasty and total shoulder arthroplasty in patients aged fifty years or younger. J Shoulder Elbow Surg. 2004;13:604-613.

38. Spiegl UJ, Faucett SC, Horan MP, Warth RJ, Millett PJ. The role of arthroscopy in the management of glenohumeral osteoarthritis: a Markov decision model. Arthroscopy. 2014;30:1392-1399.

39. US Census Bureau. 2012 Population Estimate. Available at: http://www.census.gov/population/age/data/files/2012/2012gender_ table1.xlsx. Accessed June 27, 2016.

40. Weinstein MC. How much are Americans willing to pay for a quality-adjusted life year? Med Care. 2008;46:343-345. 